\title{
Spectrometric Characterization of Amorphous Silicon PIN Detectors
}

\author{
Leyva A. , Ramírez F.J. ${ }^{2}$, Ortega Y. ${ }^{1}$, Estrada M. ${ }^{3}$, Cabal A.', \\ Cerdeira A. ${ }^{3}$, Díaz A. ${ }^{1}$ \\ ${ }^{\prime}$ Centro de Estudio Aplicados al Desarrollo Nuclear, P.O.Box 6122, La Habana, Cuba \\ ${ }^{2}$ Instituto Nacional de Investigaciones Nucleares, P.O. Box 19-1027, México D. F., México. \\ ${ }^{3}$ Sección de Electrónica del Estado Sólido, Depto. de Ingeniería Eléctrica, CINVESTAV-IPN, \\ P.O.Box 14740 , Mexico D.F., Mexico.
}

\begin{abstract}
During the last years, much interest has been dedicated to the use of amorphous silicon PIN diodes as particle and radiation detectors for medical applications. This work presents the spectrometric characterization of PECVD high deposition rate diodes fabricated at our laboratory, with thickness up to $17.5 \mu \mathrm{m}$. Results show that the studied devices detect the $\mathrm{Am}^{241}$ alpha particles and the medical X-rays generated by a mammograph model Senographe $700 \mathrm{~T}$ from General Electric. Possible reasons of the observed energy losses are discussed in the 1ext. Using the SRIM2000 program, the transit of $5.5 \mathrm{MeV}$ alpha particles through a diode was simulated, determining the optimum thickness for these particles to deposit their energy in the intrinsic layer of the diode.
\end{abstract}

\section{INTRODUCTION}

Much effort is being dedicated to the research of amorphous silicon PIN diodes for applications as detectors for image generation in nuclear medicine $[1,2]$ because of a low production cost, high stability to radiation damage and the possibility to grow large active areas. These devices can also be integrated with their read-out circuits. An array of a-Si:H pin photodiodes in conjunction with a scintillation film is already commercially available [3] for substituting the X-ray screen-films in medical radiography. This method of detection is usually called indirect detection. A direct detection of X-rays by these amorphous PIN diodes will provide better resolution among other advantages. However, the deposition of thick layers in a reasonable time, with good electronic characteristics, stability and repeatability is still under development. Previously, we reported a high deposition rate technique developed in our laboratory [4], as well as the electrical characterization of layers up to $17.5 \mu \mathrm{m}$ thick [5], that demonstrate the good quality of the deposited films. In this work we report the spectrometric characterization of devices fabricated on these films. 


\section{EXPERIMENTAL DETAILS}

The amorphous PIN diodes characterized in this work have been fabricated by the plasma enhancement chemical vapor deposition method (PECVD), using the high deposition rate reported in [4].

Two types of diodes were characterized: diodes marked as DA had an intrinsic aSi:H layer $17.5 \times 10^{-4} \mathrm{~cm}$ thick; a $\mathrm{Cr}$ layer $0.5 \times 10^{-4} \mathrm{~cm}$ thick and an area equal to 65 $\times 10^{-4} \mathrm{~cm}^{2}$. Diodes named EN had an intrinsic layer $1.7 \times 10^{-4} \mathrm{~cm}$ thick; a Cr layer $1 \mathrm{x}$ $10^{-4} \mathrm{~cm}$ thick and an area equal to $4 \times 10^{-4} \mathrm{~cm}^{2}$.

The detector is reverse biased to achieve an electric field across the whole i-layer, with a minimum value of at least $1.2 \times 10^{4} \mathrm{~V} / \mathrm{cm}$ at $\mathrm{i}-\mathrm{n}^{+}$borders. For alpha particle detection, the detector is connected to a low noise charge sensitive preamplifier as described in [6]. The optimized shaping time was set to $1.5 \mu \mathrm{s}$. The spectral response is recorded by a multichannel analyzer. All measurements took place at room temperature. The natural radioisotope $\mathrm{Am}^{241}$ was used as source. For the detection of $\mathrm{X}$-rays, diodes were irradiated with a model Senographe 700T General Electric mammograph. The X-ray tube produces a photon flux with mean energy of $8.7 \mathrm{KeV}$.

\section{RESULTS AND DISCUSSION}

Curves $\triangle$ in figure 1 show the experimental spectra obtained for DA diodes with a reverse bias of $200 \mathrm{~V}$ (a) and for EN diodes with $10 \mathrm{~V}$ of reverse bias (b), when exposed to a $1 \mu \mathrm{Ci} \mathrm{Am}^{241}$ alpha source. Noise spectra when diodes are not exposed to radiation are indicated in figures by dots.

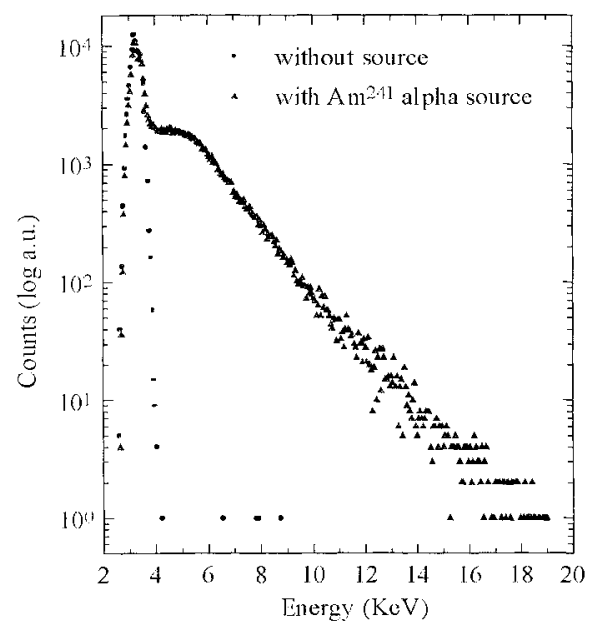

(a)

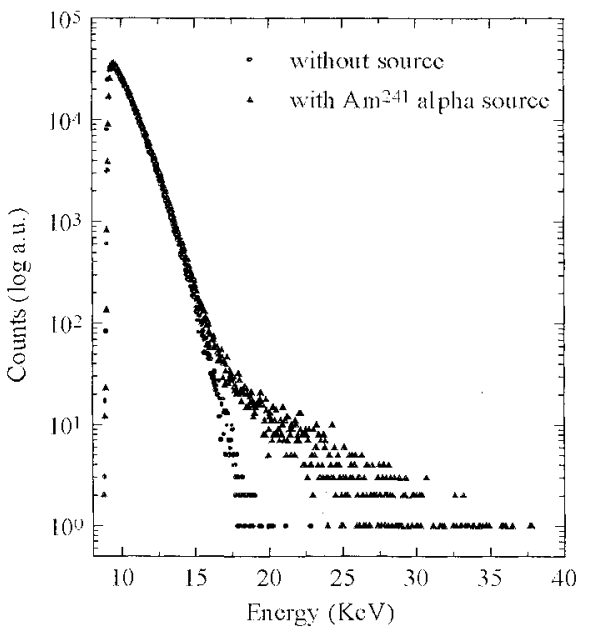

(b)

FIGURE 1. Experimental spectra obtained for DA diodes (a) and EN diodes (b) when exposed to an $\mathrm{Am}^{2 \cdot 11}$ alpha source $₫$ and without source $\bullet$. 


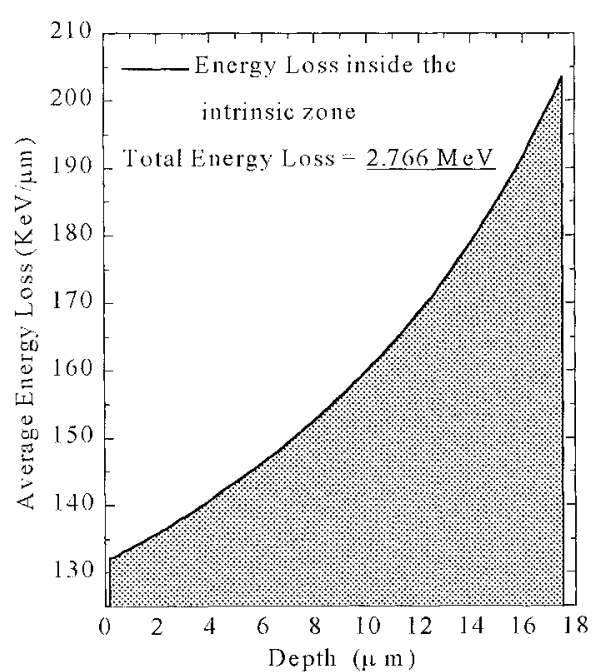

(a)

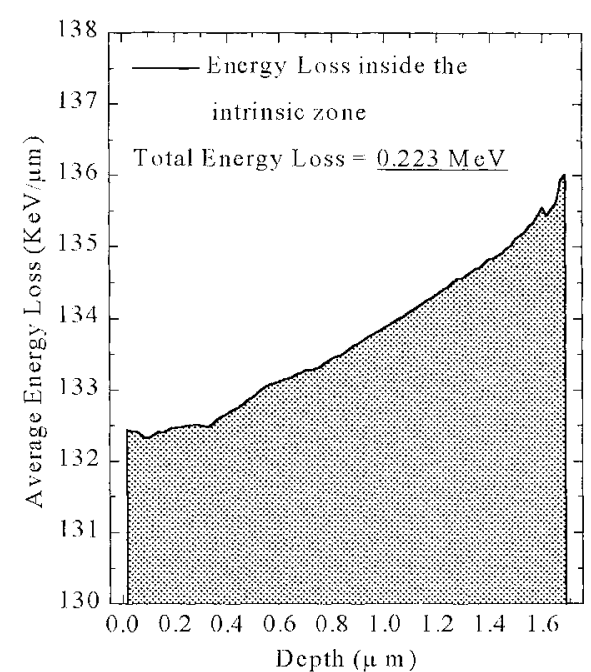

(b)

FIGURE 2. Average energy loss of alpha particles while traveling across the i-layer of diode DA (a) and diode EN (b).

The energy spectrum of DA diode shows a very wide peak with maximum at 5 $\mathrm{KeV}$. The left part of the peak is overlapped by background noise while the right extends up to energies near $20 \mathrm{KeV}$. EN diodes show a very high background noise which difficults the observation of the radiation response, although in the obtained spectrum the tail extending for energies greater than $17 \mathrm{KeV}$ indicates particle registration. For EN diodes, no peak was observed in the entire spectrum.

To compare with the experiment, the transit of the $5.5 \mathrm{MeV}$ alpha particle through the diodes was simulated using the software SRIM2000 [7]. Real dimensions of each device layer were taken into account. Figure 2 (a) shows the simulated behavior of the energy loss of an alpha particle as it moves inside the intrinsic zone of diode DA. It was calculated that the particles trespassing air $(0.2 \mathrm{~cm}), \mathrm{Cr}$ electrode and $\mathrm{p}$ doped $\mathrm{Si}$ layer, enter the i-layer with an energy of $5.32 \mathrm{MeV}$. Inside the $17.5 \mu \mathrm{m}$ thick i-layer, they will deposit only $2.77 \mathrm{MeV}$. Figure 2 (b) shows that for diodes EN, only 0.223 $\mathrm{MeV}$ of the $5.32 \mathrm{MeV}$ will be deposited in the i-layer. The i-layer thickness to stops completely the alpha particles resulted $28.5 \mu \mathrm{m}$.

Considering the simulation results, the experiment indicates there is still less collection than expected. This could be attributed to a strong recombination process due to a plasma effect that is induced by the high charge density generated by the high ionizing alpha particles, favored by the working temperature. Also the small carrier mobility can make the collection process very slow so only a small fraction of carriers contribute to the signal when using the selected shaping time of few microseconds.

Finally, since amorphous materials have a very high defect density, these defects can act as traps for carriers [8] giving a reason to presume that a significant percent of the generated carriers are trapped within a few microseconds and detrapped at times much longer than the shaping time. For this reason they will not be detected. 


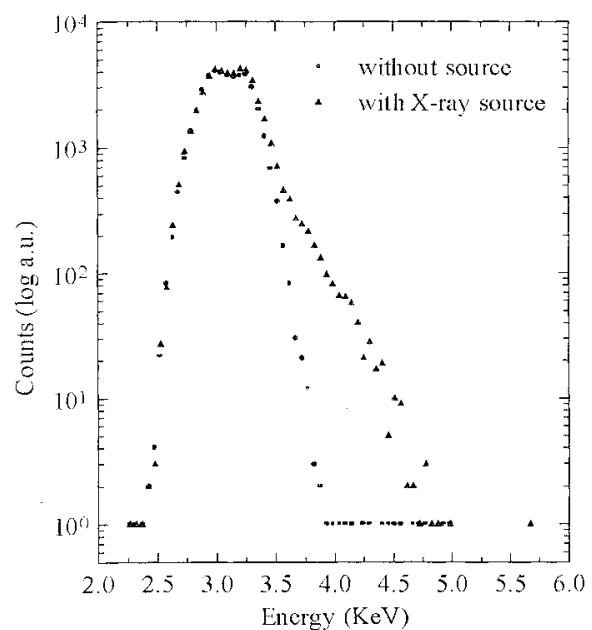

(a)

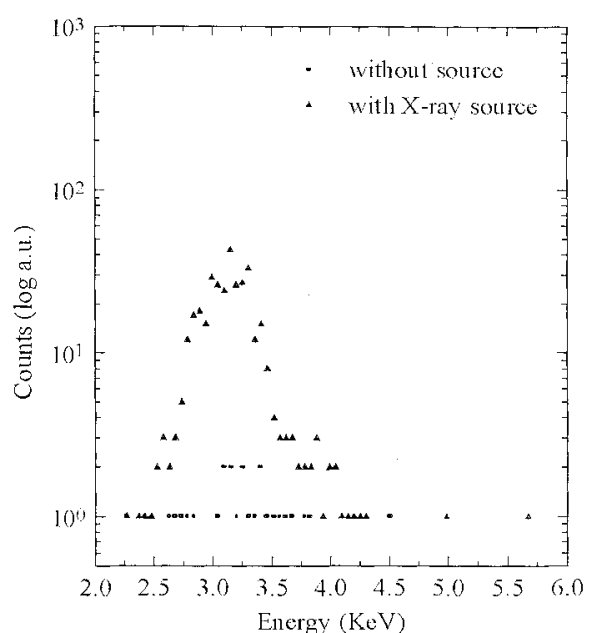

(b)

FIGURE 3. Experimental spectra obtained for $200 \mathrm{~V}$ reverse biased DA diodes (a) and $300 \mathrm{~V}$ reverse biased DA diodes (b), when exposed to the X-rays generated by a commercial mammograph $\Delta$, and without source $\bullet$.

The response of diodes to the mammograph X-rays if any, was expected only for the thicker diodes DA. For this measurement we used molybdenum X-ray tube of the above-indicated mammograph biased at $25 \mathrm{KV}$. Current was fixed at $4 \mathrm{~mA}$-s.

In figure 3 (a) a spectrum measured with $200 \mathrm{~V}$ reverse biased diode is observed. Despite a high noise, the sensibility of the diode to the radiation is obvious. With the irradiation, a bulk appears in the right side of the peak. Increasing the polarization of the diode up to $300 \mathrm{~V}$, the noise background drops down as seen in figure 3 (b). The before observed bulkiness now transforms into a clear peak with the maximum positioned at $3.20 \mathrm{KeV}$.

When the current in the X-ray tube is increased, which is similar to an increment of the exposure time, an improvement of the signal to noise ratio was observed.

\section{CONCLUSION}

High deposition rate PIN type a-Si:H diodes were characterized when exposed to the $\mathrm{Am}^{241}$ alpha source and to X-rays generated by a commercial mammograph. The recorded spectra for the alpha source show very intense energy loss indicating that the diodes were not optimized for this kind of radiation. The loss and poor collection efficiency should be related with the physical processes present in the material as plasma effect due to the alpha particle interaction, recombination and trapping phenomena increased by the presumable low material quality. The thickness of intrinsic diode layers is not sufficient to guaranty the maximal deposition energy of the particles; it should be $28.5 \mu \mathrm{m}$. In the case of diodes irradiation by mammograph X- 
rays, higher detection efficiency was observed fundamentally when the diodes where $300 \mathrm{~V}$ reverse biased.

\section{ACKNOWLEDGMENTS}

Authors would like to thank the Ionizing Radiation Metrology Laboratory of ININ for the use of his standards, $\mathrm{X}$-ray units and installations. This work was supported by CONACYT project $28092 \mathrm{~A}$.

\section{REFERENCES}

1. Aflatooni $\Lambda$, Nathan A., Fornsey R., Conningham I., and Chamberlain S., "a-Si:H Schottky diode direct detection pixel for large area X-ray imaging", Technical Digest of the HEDM, Washington D.C. December 7 10, pp. $197-200,(1997)$

2. Estrada M. and Cabal A., "Medical application of amorphous silicon detectors", Medical Physic: Second Mexican Symposium, edited by Brandan M. E., Ortega R. and Herrera G., AlP Conference Proceeding, pp. 179 $-186,(1998)$

3. Weisfield R., "Amorphous silicon TFT X-ray image sensors", Technical Digest of the LLDM, Washington D.C. December 6-9, pp. $21-24,(1998)$.

4. Soto S., Estrada M., Merkulov A and Asomoza R., "High deposition rate of amorphous silicon thick layers using a gas mixture of $10 \%$ of silane in hydrogen", Thin Solid Films, 330, p. $83-88,(1998)$

5. Estrada M., Cerdeira A., Pereyra I. and Soto S., "Characteristics of high deposition rate pin diodes from pure $\mathrm{SiH}_{4}$ and $10 \%$ dissolution of $\mathrm{SiH}_{4}$ in $\mathrm{H}_{2}{ }^{\prime}$, to be published in IEEE Trans. Nucl. Sci., 47, No. 1, (2000).

6. Ramírez F. J., Díaz A., Cerdeira A., Leyva A., Montaño L. M., Estrada M., "Low noise charge sensitive preamplifier for the measurement of low energy and X-rays with pin diode", Proceeding of the XXII National Symposium of Biomedical Engine, Ixtapa-Zihuatanejo, November 10-12, (1999).

7. www.research.ibm.com/ionbeams/

8. Crandall R. S., "Photoconductivity", Semiconductors and Semimetals, 21, part B, pp. $245-297,(1984)$. 